\title{
J-Integral Solution for Elastic Fracture Toughness for Plates with Inclined Cracks under Biaxial Loading
}

\author{
Chun-Qing $\mathrm{Li}^{1}$, Guoyang $\mathrm{Fu}^{1}$, Wei Yang ${ }^{2}$ and Shangtong Yang ${ }^{3}$
}

\begin{abstract}
Surface cracks with different orientations have been recognized as a major cause of potential failures of thin metal structures, which are often under biaxial loading. It has been known that, for cracked ductile metals, plasticity results in an easing of stress intensity at the crack front and ultimately increases the total fracture toughness of the metal. To enable the use of linear elastic fracture mechanics for ductile material failure prediction, the plastic portion of fracture toughness must be excluded. This paper aims to develop a $J$-integral based method for determining the elastic fracture toughness of ductile metal plates with inclined cracks under biaxial loading. The derived elastic fracture toughness is a function of the plate and crack geometry, strain-hardening coefficient, yield strength, fracture toughness, biaxiality ratio, and inclination angle. It is found that an increase in yield strength or relative crack depth, or a decrease in Mode-I fracture toughness, leads to a larger ratio of elastic fracture toughness to total fracture toughness. It is also found that the effect of biaxiality ratio and inclination angle on elastic fracture toughness is highly dependent on total fracture toughness. It can be concluded that the developed model can accurately predict the fracture failure of ductile thin metal structures with inclined cracks under biaxial loading.
\end{abstract}

Author keywords: Inclined surface cracks; J-integral; Biaxial loading; Elastic fracture toughness; Cracked plates.

\section{Introduction}

Surface cracks, which may appear in different orientations, have long been recognized as a major cause of potential failure in structures made of ductile metals. More often than not, these structures are subjected to biaxial loading caused by thermal stress, pressure, and/or other external loads. A plate with an inclined surface crack under biaxial loading (Fig. 1) is a typical model of biaxially loaded structural components which is of significant practical importance for engineering assessment. Methodologies for assessing cracked structure failure are relatively well-established (Anderson 1991); they require estimation of crack driving force (e.g., stress intensity factor, $J$-integral) and corresponding fracture toughness.

Mode-I fracture toughness experimentally measured under uniaxial loading has traditionally been considered the conservative limit. However, some ductile materials have been found to have lower fracture toughness under biaxial loadings for Mode-I fracture (Bass et al. 1992) or mixed-mode loadings (Kamat and Hirth 1995). For effects of biaxial loadings on fracture toughness, exper-imental studies have been conducted by researchers (Jones et al. 1986; Bass et al. 1996; Mostafavi et al. 2011) on center-cracked specimens with different biaxiality ratios, defined as the ratio of two perpendicular applied stresses. A literature review revealed that mixed-mode fracture toughness is determined by taking into account the effects of either Mode-II (e.g., Keiichiro and Hitoshi 1992; Kamat and Hirth 1996; Hallbäck 1997) or Mode-III loading (e.g., Manoharan et al. 1990; Kamat et al. 1994; Liu et al. 2004; Paradkar and Kamat 2011) or Modes-II and III loadings (Richard and Kuna 1990; Richard et al. 2013) on the total fracture toughness of brittle and ductile materials.
It has been known that plasticity increases fracture toughness. The underlying mechanism is that yielding caused by plasticity eases stress concentration at the crack front. Consequently, fracture toughness increases and consists of elastic and plastic portions. In order to extract elastic fracture toughness from total fracture toughness, a failure assessment diagram was employed by Yang et al. (2016, 2017) and Li et al. (2017). The adopted failure assessment curve (Milne et al. 1988) is independent of both geometry and material properties and may be used for any structure. However, the derived elastic fracture toughness models may be overconservative, given that the curve was derived as a lower bound of the failure assessment diagrams obtained based on reference stress (SINTAP 1999). An alternative to the failure assessment diagram is the $J$-integral. Based on separation of elastic and plastic displacements, the $J$-integral can naturally be separated into elastic and plastic components (Zhu and Joyce 2012). When the applied load reaches its critical value, the elastic $J$-integral corresponds to elastic fracture toughness. Compared with the failure assessment diagram, the $J$-integral is more rigorous, allowing more accurate elastic fracture toughness models to be developed.

The aim of this paper is to develop a $J$-integral-based method to determine elastic fracture toughness for plates with inclined surface cracks subjected to biaxial loadings. The $J$-integral is proposed in this paper for separation of elastic fracture toughness from total fracture toughness. Because the $J$-integral uniquely characterizes the crack tip field in nonlinear materials, the accuracy of the developed elastic fracture toughness models can be guaranteed. The derived elastic fracture toughness is a function of plate and crack geometry, strain-hardening coefficient, yield strength, fracture toughness, biaxiality ratio, and inclination angle. After verification of the developed model for elastic and fully plastic $J$-integrals,

\footnotetext{
${ }^{1}$ School of Engineering, RMIT Univ., Melbourne 3000, Australia.

${ }^{2}$ College of Engineering and Science, Victoria Univ., Melbourne, Australia.

${ }^{3}$ Department of Civil and Environmental Engineering, University of Strathclyde, United Kingdom.
} 


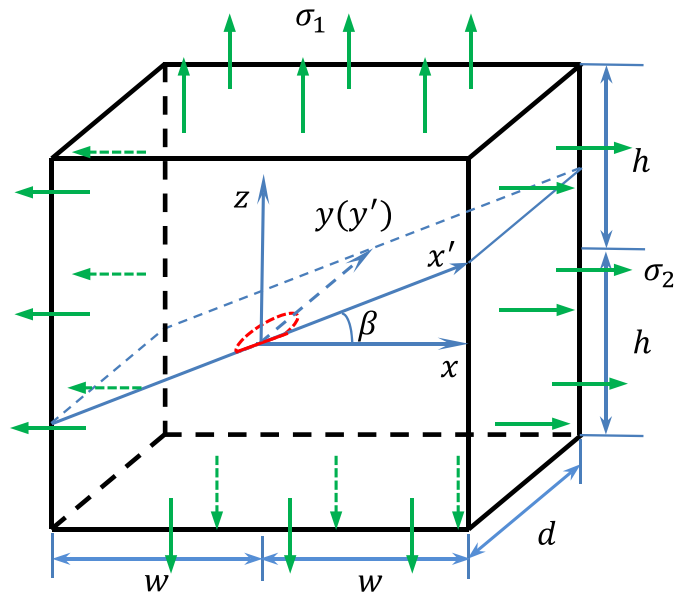

(a)

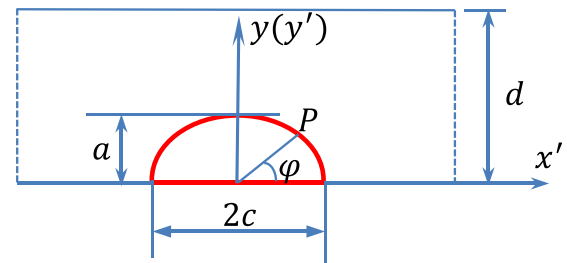

(b)

Fig. 1. Inclined surface crack in a plate under biaxial loading

parametric studies are conducted to investigate the effect of key parameters on elastic fracture toughness. The significance of the study is to allow the use of linear elastic fracture mechanics and associated results by engineers and asset managers for both design and assessment of cracked plates made of ductile metals.

\section{Theory of Elastic Fracture Toughness}

It is known that in linear elastic fracture mechanics a cracked structure fails when stress intensity factor $K$ at the crack front exceeds fracture toughness $K_{C}$. For ductile materials, plasticity develops at the crack front, which eases stress concentration. To take into account the effect of plasticity, the stress intensity factor for a cracked structure under an applied load $F$ can be written as

$$
K=K^{e}(F)+K^{p}(F)
$$

where $K^{e}$ and $K^{p}=$ elastic and plastic components of the stress intensity factors, respectively, and both are a function of applied load $F$. The critical stress intensity factor (i.e., fracture toughness $K_{C}$ ) is determined by a critical load $F_{C}$ corresponding to the instability point (crack initiation). As a result, fracture toughness can be expressed as follows:

$$
K_{C}=K_{C}^{e}\left(F_{C}\right)+K_{C}^{p}\left(F_{C}\right)
$$

Because $K_{C}$ is a material constant, the determination of $K_{C}^{e}$ means that $K_{C}^{p}$ is known. In Eq. $(1), K^{e}(F)$ is effectively the stress intensity factor for brittle materials. For given cracked structures, when $K^{e}$ reaches $K_{C}^{e}, K^{p}$ reaches $K_{C}^{p}$. Therefore, the failure criterion for ductile materials from Eq. (2) can be changed to the following:

$$
K^{e} \geq K_{C}^{e}
$$

It is implied that linear elastic fracture mechanics can be employed once elastic fracture toughness $K_{C}^{e}$ is determined. However, $K_{C}^{e}$ does not mean that the plastic influences are omitted. On the contrary, the effects of plasticity are taken into consideration in the derivation of elastic fracture toughness.

The failure assessment diagram method may produce overconservative results; therefore, in this paper the $J$-integral method is used to determine elastic fracture toughness.

\section{Derivation of Elastic Fracture Toughness}

The $J$-Integral is a path-independent contour integral for the analysis of stresses around cracks (Rice 1968). It uniquely characterizes crack tip stresses and strains in nonlinear materials (Hutchinson 1968; Rice and Rosengren 1968). According to Zhu and Joyce (2012), for ductile materials the $J$-integral consists of both elastic and plastic portions, which can be represented as follows:

$$
J=J_{e}+J_{p}
$$

where $J_{e}$ and $J_{p}=$ elastic and plastic $J$-integrals, respectively.

For evaluation of the $J$-integral, the Electric Power Research Institute (EPRI) method based on deformation plasticity (Kumar et al. 1981) is the most widely used because it calculates the $J$-integral from an elastic plastic condition up to a fully plastic condition (Kumar et al. 1981). In the EPRI method, the total $J$-integral is calculated by adding up its elastic and fully plastic portions (Kumar et al. 1981)

$$
J=J_{e}\left(a_{e f f}\right)+J_{f p}
$$

where $J_{e}$ and $J_{f p}=$ elastic and fully plastic $J$-integral, respectively. A plastic zone correction is considered for the calculation of $J_{e}$ to obtain a better estimate of the $J$-integral (Kumar et al. 1981), after which $a_{\text {eff }}=$ crack depth and can be determined by the following equation (Sih 1976):

$$
\begin{gathered}
a_{e f f}=a+r_{y} \\
r_{y}=\frac{1}{m}\left(\frac{n-1}{n+1}\right)\left(\frac{K_{e q}}{\sigma_{y}}\right)^{2}
\end{gathered}
$$

where $a=$ crack depth; $m=2$ for plane stress problems; $m=6$ for plane strain problems; $n=$ strain-hardening exponent; $\sigma_{y}=$ yield stress; and $K_{e q}=$ equivalent stress intensity factor, expressed as follows:

$$
K_{e q}=\sqrt{K_{I}^{2}+K_{I I}^{2}+(1+v) K_{I I I}^{2} E^{\prime} / E}=\sigma_{1} \sqrt{\frac{\pi a}{Q}} F_{e q}
$$

where $K_{I}, K_{I I}, K_{I I I}=$ stress intensity factors for Modes I, II, and III, respectively; and $v=$ Poisson's ratio; for plane strain problems, $E^{\prime}$ needs to be replaced by $E /\left(1-v^{2}\right)$ whereas for plane stress problems, $E^{\prime}=E ; \sigma_{1}=$ applied stress; $a=$ crack depth; 
$F_{e q}=$ equivalent influence coefficient, which is a function of the geometry of the cracked plate; and $Q=$ shape factor for an ellipse and is given by the square of the complete elliptical integral of the second kind (Green and Sneddon 1950). The shape factor $Q$ is approximated in practical applications by the following empirical formula (Shiratori et al. 1987):

$$
Q=1+1.464\left(\frac{a}{c}\right)^{1.65} \frac{a}{c} \leq 1
$$

where $c=$ half of the crack length.

The elastic $J$-integral $J_{e}$ is related to the stress intensity factors as follows (Anderson 2005):

$$
J_{e}=\left(K_{e q}\right)^{2} / E^{\prime}
$$

With the Ramberg-Osgood stress strain relationship (Ramberg and Osgood 1943), the fully plastic $J$-integral $J_{f p}$ of cracked plates under biaxial loading can be expressed as follows (Jansson 1986):

$$
J_{f p}=J-J_{e}=h_{1} \alpha \sigma_{y} \varepsilon_{y} d\left(\frac{\sigma_{n e}}{\sigma_{y}}\right)^{n-1}\left(\frac{\sigma_{1}}{\sigma_{y}}\right)^{2}
$$

where $\alpha=$ material constant; $\varepsilon_{y}=$ strain with a value of $\sigma_{y} / E ; h_{1}=$ normalized fully plastic $J$-integral, which is a function of $a / c, a / d$, $\varphi, n$, and $\beta ; d=$ plate thickness; $\varphi=$ position of Point $\mathrm{P}$ along the crack front as shown in Fig. 1 ; and $\sigma_{n e}=$ nominal von Mises equivalent stress, defined as follows:

$$
\sigma_{n e}=\sqrt{\sigma_{1}^{2}+\left(\lambda \sigma_{1}\right)^{2}-\sigma_{1}\left(\lambda \sigma_{1}\right)}=\sigma_{1} \sqrt{1-\lambda+\lambda^{2}}
$$

where $\lambda=$ biaxiality ratio, defined as the ratio of the stress $\sigma_{1}$ along the $z$-axis to the stress $\sigma_{2}$ along the $x$-axis (Fig. 1)

$$
\begin{aligned}
J & =\left[\sigma_{1} \sqrt{\frac{\pi a_{\mathrm{eff}}}{Q}} F_{e q}\left(a_{\mathrm{eff}}\right)\right]^{2} / E^{\prime}+h_{1} \alpha \sigma_{y} \varepsilon_{y} d\left(\frac{\sigma_{n e}}{\sigma_{y}}\right)^{n-1}\left(\frac{\sigma_{1}}{\sigma_{y}}\right)^{2} \\
& =J_{C}=\frac{\left(K_{C}\right)^{2}}{E^{\prime}}
\end{aligned}
$$

When $\sigma_{1}$ reaches a critical value $\sigma_{1 c}$, the $J$-integral becomes the crack initiation fracture toughness $J_{C}$. By rearranging Eq. (13), the following equation is obtained:

$$
\begin{aligned}
& \frac{\left[\sqrt{\frac{\pi a_{\text {eff }}}{Q}} F_{e q}\left(a_{\text {eff }}\right)\right]^{2}}{E^{\prime}} *\left(\sigma_{1}\right)^{2}+\frac{h_{1} \alpha \sigma_{y} \varepsilon_{y} d\left(\sqrt{1-\lambda+\lambda^{2}}\right)^{n-1}}{\left(\sigma_{y}\right)^{n+1}}\left(\sigma_{1}\right)^{n+1} \\
& =J_{C}=\frac{\left(K_{C}\right)^{2}}{E^{\prime}}
\end{aligned}
$$

Elastic fracture toughness can then be calculated by the following equation:

$$
K_{C}^{e}=\sigma_{1 c} \sqrt{\frac{\pi a}{Q}} F_{e q}
$$

Accordingly, plastic fracture toughness $K_{C}^{p}$ can be determined as follows:

$$
K_{C}^{p}=K_{C}-K_{C}^{e}
$$

\section{J-Integral and Mixed-Mode Fracture Toughness}

From Eqs. (14) and (15), it can be seen that for a given cracked plate the main effort of determining elastic fracture toughness lies in the calculation of the elastic $J$-integral-that is, the equivalent influence coefficients $F_{e q}$, the normalized fully plastic $J$-integral $h_{1}$, and the mixed-mode fracture toughness $J_{C}\left(K_{C}\right)$.

\section{Equivalent Influence Coefficients}

By substituting Eq. (8) into Eq. (10), the equivalent influence coefficient $F_{e q}$ is related to the elastic $J$-integral by the following equation:

$$
F_{e q}=\sqrt{\frac{J_{e} Q E^{\prime}}{\pi a \sigma_{1}^{2}}}
$$

To determine the equivalent influence coefficients for cracked plates with inclined surface cracks, three-dimensional (3D) finiteelement fracture analyses were performed by $\mathrm{Fu}$ et al. (2017a) using $A B A Q U S$. In the finite-element models, a conformed mesh was used around the crack region. A meshing technique with mixed quadratic hexahedron and tetrahedron elements in $\mathrm{Li}$ et al. (2016) and Fu et al. (2017b) was adopted. In addition, the energybased $J$-integral method, which is path-independent and can produce accurate results with relatively coarse meshes, was employed. Because of the free surface effect (Pook 1995), $J$-integral values at the surface points were estimated by extrapolation from the results close to the surface.

The equivalent influence coefficients $F_{e q}$ for inclined cracks in plates under biaxial loading were determined. Table 1 shows $F_{e q}$ for different relative crack depth ratios $a / d$, biaxiality ratios $\lambda$, and inclination angles $\beta$. The parameter $\varphi$ denotes the position of a point along the semielliptical surface crack as shown in Fig. 1.

\section{Normalized Fully Plastic J-Integral}

Based on the deformation theory of plasticity, the same finiteelement models used for the equivalent influence coefficients were adopted for determining fully plastic $J$-integral values ( $\mathrm{Fu}$ et al. 2017a). These values for plates with different relative crack depths, biaxiality ratios, inclination angles, and strain-hardening exponents were determined. The normalized $J$-integral $h_{1}$ values were calculated using the following equation:

$$
h_{1}=\frac{J-J_{e}}{\alpha \sigma_{y} \varepsilon_{y} d\left(\frac{\sigma_{n e}}{\sigma_{y}}\right)^{n-1}\left(\frac{\sigma_{1}}{\sigma_{y}}\right)^{2}}
$$

Table 2 lists the $h_{1}$ values for strain-hardening coefficient $n=3$. For different values of $n$ and negative biaxiality ratios, see Fu et al. (2017a) for details.

\section{Fracture Toughness of Ductile Materials}

It has been known that some ductile-material fracture toughness values are affected by both biaxial loading for Mode-I fracture (Bass et al. 1992) and mixed-mode loadings (Kamat and Hirth 1995).

Jones et al. (1986) tested flat-sheet center-cracked specimens over a wide range of biaxiality ratios for different materials and found that the fracture toughness of two aluminum alloys reaches peak values at a biaxiality ratio of 0.5 and then decreases as that ratio increases. Bass et al. $(1992,1996)$ showed that a reduction in fracture toughness of up to $40 \%$ in biaxially loaded specimens compared with uniaxially loaded specimens. Mostafavi et al. (2011) carried out biaxial tests on 2024 aluminum alloy and showed that 
Table 1. Equivalent Influence Coefficients $F_{e q}$

\begin{tabular}{|c|c|c|c|c|c|c|c|c|c|c|}
\hline \multirow[b]{2}{*}{$\beta$ (degrees) } & \multirow[b]{2}{*}{$\varphi$ (degrees) } & \multicolumn{3}{|c|}{$a / d=0.2$} & \multicolumn{3}{|c|}{$a / d=0.5$} & \multicolumn{3}{|c|}{$a / d=0.8$} \\
\hline & & $\lambda=0$ & $\lambda=0.5$ & $\lambda=1$ & $\lambda=0$ & $\lambda=0.5$ & $\lambda=1$ & $\lambda=0$ & $\lambda=0.5$ & $\lambda=1$ \\
\hline \multirow[t]{8}{*}{0} & 0 & 1.167 & 1.167 & 1.167 & 1.255 & 1.255 & 1.255 & 1.420 & 1.420 & 1.420 \\
\hline & 10 & 1.144 & 1.144 & 1.144 & 1.232 & 1.232 & 1.232 & 1.375 & 1.375 & 1.375 \\
\hline & 20 & 1.103 & 1.103 & 1.103 & 1.178 & 1.178 & 1.178 & 1.294 & 1.294 & 1.294 \\
\hline & 38 & 1.067 & 1.067 & 1.067 & 1.124 & 1.124 & 1.124 & 1.200 & 1.200 & 1.200 \\
\hline & 51 & 1.054 & 1.054 & 1.054 & 1.102 & 1.102 & 1.102 & 1.153 & 1.153 & 1.153 \\
\hline & 64 & 1.047 & 1.047 & 1.047 & 1.088 & 1.088 & 1.088 & 1.120 & 1.120 & 1.120 \\
\hline & 77 & 1.042 & 1.042 & 1.042 & 1.080 & 1.080 & 1.080 & 1.097 & 1.097 & 1.097 \\
\hline & 90 & 1.040 & 1.040 & 1.040 & 1.076 & 1.076 & 1.076 & 1.089 & 1.089 & 1.089 \\
\hline \multirow[t]{8}{*}{25} & 0 & 1.075 & 1.082 & 1.167 & 1.164 & 1.174 & 1.255 & 1.302 & 1.350 & 1.455 \\
\hline & 10 & 1.040 & 1.065 & 1.144 & 1.108 & 1.144 & 1.232 & 1.222 & 1.274 & 1.375 \\
\hline & 20 & 1.006 & 1.028 & 1.103 & 1.065 & 1.096 & 1.178 & 1.158 & 1.201 & 1.295 \\
\hline & 38 & 0.974 & 0.994 & 1.067 & 1.019 & 1.046 & 1.124 & 1.083 & 1.117 & 1.202 \\
\hline & 51 & 0.959 & 0.982 & 1.054 & 0.997 & 1.025 & 1.102 & 1.042 & 1.073 & 1.154 \\
\hline & 64 & 0.948 & 0.974 & 1.047 & 0.981 & 1.011 & 1.088 & 1.013 & 1.042 & 1.121 \\
\hline & 77 & 0.941 & 0.969 & 1.042 & 0.970 & 1.002 & 1.079 & 0.993 & 1.022 & 1.098 \\
\hline & 90 & 0.938 & 0.967 & 1.040 & 0.966 & 0.999 & 1.075 & 0.985 & 1.014 & 1.090 \\
\hline \multirow[t]{8}{*}{45} & 0 & 0.864 & 0.931 & 1.167 & 0.918 & 1.010 & 1.255 & 0.992 & 1.142 & 1.420 \\
\hline & 10 & 0.815 & 0.906 & 1.144 & 0.853 & 0.970 & 1.232 & 0.917 & 1.075 & 1.375 \\
\hline & 20 & 0.794 & 0.875 & 1.102 & 0.827 & 0.930 & 1.178 & 0.881 & 1.016 & 1.295 \\
\hline & 38 & 0.769 & 0.847 & 1.066 & 0.795 & 0.889 & 1.124 & 0.836 & 0.947 & 1.201 \\
\hline & 51 & 0.753 & 0.835 & 1.054 & 0.775 & 0.870 & 1.102 & 0.809 & 0.911 & 1.155 \\
\hline & 64 & 0.739 & 0.827 & 1.047 & 0.758 & 0.858 & 1.088 & 0.787 & 0.886 & 1.122 \\
\hline & 77 & 0.729 & 0.822 & 1.042 & 0.746 & 0.850 & 1.079 & 0.773 & 0.869 & 1.100 \\
\hline & 90 & 0.724 & 0.820 & 1.040 & 0.742 & 0.847 & 1.076 & 0.766 & 0.862 & 1.092 \\
\hline
\end{tabular}

Table 2. Normalized Fully Plastic $J$-Integral $h_{1}$ for $n=3$

\begin{tabular}{|c|c|c|c|c|c|c|c|c|c|c|}
\hline \multirow[b]{2}{*}{$\beta$ (degrees) } & \multirow[b]{2}{*}{$\varphi$ (degrees) } & \multicolumn{3}{|c|}{$a / d=0.2$} & \multicolumn{3}{|c|}{$a / d=0.5$} & \multicolumn{3}{|c|}{$a / d=0.8$} \\
\hline & & $\lambda=0$ & $\lambda=0.5$ & $\lambda=1$ & $\lambda=0$ & $\lambda=0.5$ & $\lambda=1$ & $\lambda=0$ & $\lambda=0.5$ & $\lambda=1$ \\
\hline \multirow[t]{8}{*}{0} & 0 & 0.209 & 0.191 & 0.157 & 0.669 & 0.559 & 0.442 & 1.772 & 1.521 & 1.172 \\
\hline & 10 & 0.369 & 0.398 & 0.353 & 1.287 & 1.258 & 1.079 & 2.909 & 2.756 & 2.277 \\
\hline & 20 & 0.377 & 0.414 & 0.358 & 1.301 & 1.300 & 1.088 & 2.872 & 2.791 & 2.266 \\
\hline & 38 & 0.376 & 0.393 & 0.331 & 1.247 & 1.207 & 0.981 & 2.560 & 2.463 & 1.950 \\
\hline & 51 & 0.372 & 0.365 & 0.306 & 1.202 & 1.099 & 0.887 & 2.299 & 2.122 & 1.663 \\
\hline & 64 & 0.369 & 0.333 & 0.281 & 1.152 & 0.981 & 0.792 & 2.031 & 1.783 & 1.399 \\
\hline & 77 & 0.366 & 0.313 & 0.265 & 1.117 & 0.901 & 0.732 & 1.895 & 1.618 & 1.262 \\
\hline & 90 & 0.365 & 0.305 & 0.259 & 1.100 & 0.867 & 0.708 & 1.849 & 1.575 & 1.226 \\
\hline \multirow[t]{8}{*}{25} & 0 & 0.178 & 0.157 & 0.148 & 0.588 & 0.464 & 0.454 & 1.756 & 1.698 & 1.158 \\
\hline & 10 & 0.325 & 0.336 & 0.355 & 1.064 & 1.061 & 1.099 & 2.253 & 2.306 & 2.253 \\
\hline & 20 & 0.333 & 0.350 & 0.362 & 1.067 & 1.091 & 1.109 & 2.233 & 2.321 & 2.239 \\
\hline & 38 & 0.328 & 0.331 & 0.335 & 1.015 & 1.008 & 1.001 & 2.035 & 2.055 & 1.925 \\
\hline & 51 & 0.322 & 0.306 & 0.306 & 0.969 & 0.912 & 0.895 & 1.864 & 1.783 & 1.646 \\
\hline & 64 & 0.317 & 0.284 & 0.283 & 0.930 & 0.829 & 0.809 & 1.717 & 1.550 & 1.400 \\
\hline & 77 & 0.313 & 0.269 & 0.266 & 0.900 & 0.769 & 0.747 & 1.603 & 1.386 & 1.226 \\
\hline & 90 & 0.312 & 0.264 & 0.262 & 0.886 & 0.747 & 0.724 & 1.578 & 1.350 & 1.184 \\
\hline \multirow{8}{*}{45} & 0 & 0.230 & 0.158 & 0.216 & 0.666 & 0.509 & 0.925 & 1.308 & 1.331 & 1.848 \\
\hline & 10 & 0.221 & 0.236 & 0.349 & 0.637 & 0.743 & 1.079 & 1.284 & 1.537 & 2.242 \\
\hline & 20 & 0.222 & 0.239 & 0.357 & 0.626 & 0.743 & 1.089 & 1.263 & 1.527 & 2.233 \\
\hline & 38 & 0.217 & 0.225 & 0.330 & 0.601 & 0.683 & 0.982 & 1.202 & 1.350 & 1.929 \\
\hline & 51 & 0.212 & 0.212 & 0.302 & 0.577 & 0.627 & 0.875 & 1.162 & 1.203 & 1.657 \\
\hline & 64 & 0.206 & 0.202 & 0.278 & 0.553 & 0.581 & 0.791 & 1.120 & 1.077 & 1.416 \\
\hline & 77 & 0.200 & 0.195 & 0.262 & 0.528 & 0.550 & 0.731 & 1.074 & 0.992 & 1.249 \\
\hline & 90 & 0.198 & 0.193 & 0.257 & 0.519 & 0.540 & 0.711 & 1.057 & 0.966 & 1.194 \\
\hline
\end{tabular}

fracture toughness under biaxial loading decreases by a maximum of $20 \%$ compared with that under uniaxial loading.

For the effect of mixed-mode loadings, it has been known that mixed-mode fracture toughness is dependent on material and mode mixity (Kamat and Hirth 1995). Mode mixity can be defined by the following parameter (Shih 1974):

$$
M_{e}=\frac{2}{\pi} \arctan \left(\left|\frac{K_{I}}{K_{S}}\right|\right)=\frac{2}{\pi} \arctan \sqrt{\frac{J_{I}}{J_{S}}}
$$

where $K_{S}\left(J_{S}\right)=K_{I I}\left(J_{I I}\right)$ for mixed Mode-I and Mode-II fracture and $=K_{I I I}\left(J_{I I I}\right)$ for mixed Mode-I and III fracture. For pure 

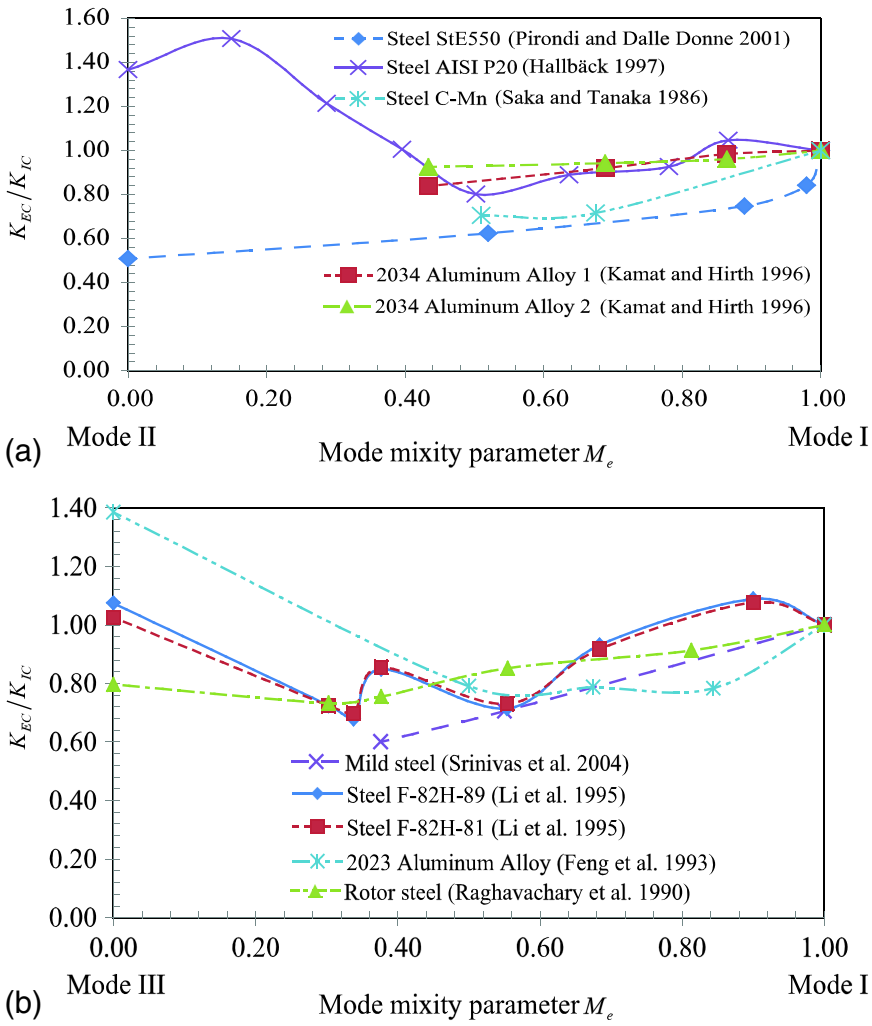

Fig. 2. Variation in normalized mixed-mode fracture toughness $K_{C} / K_{I C}$ with mode mixity parameter $M_{e}$ : (a) mixed Modes I and II; (b) mixed Modes I and III

Mode-I fracture, $M_{e}=1$; for pure Mode-II or Mode-III fracture, $M_{e}=0$.

Mixed-mode fracture toughness can be expressed as

$$
K_{C}=K_{I C} f\left(M_{e}\right)
$$

where $f=K_{C} / K_{I C}=$ function of the mode mixity parameter $M_{e}$. For a certain material, $f$ can be determined by mixed-mode experiments for various mixed modes. Mixed-mode fracture toughness is determined by taking into account the effects of either Mode-II (e.g., Keiichiro and Hitoshi 1992; Kamat and Hirth 1996; Hallbäck 1997) or Mode-III loading (e.g., Manoharan et al. 1990; Kamat et al. 1994; Liu et al. 2004; Paradkar and Kamat 2011) or Mode-II and Mode-III loadings (Richard and Kuna 1990; Richard et al. 2013) on the total fracture toughness of brittle and ductile materials. Figs. 2(a and b) shows the relationships between $f$ and $M_{e}$ determined by experiments from the literature for mixed Mode I and Mode II and mixed Mode I and Mode III, respectively. It can be seen that under mixed-mode conditions, the fracture toughness of the materials listed in Fig. 2 is generally lower than Mode-I fracture toughness. Therefore, it is essential to adopt the corresponding mixed-mode fracture toughness for assessment of mixed-mode fractures.

\section{Verification}

Before the developed elastic fracture toughness model can be applied, verification is required. Ideally, experimental results should be used for direct verification. A thorough literature review, however, reveals this to be extremely difficult. Consequently, indirect verification is employed. Regarding the EPRI method, Kumar et al.
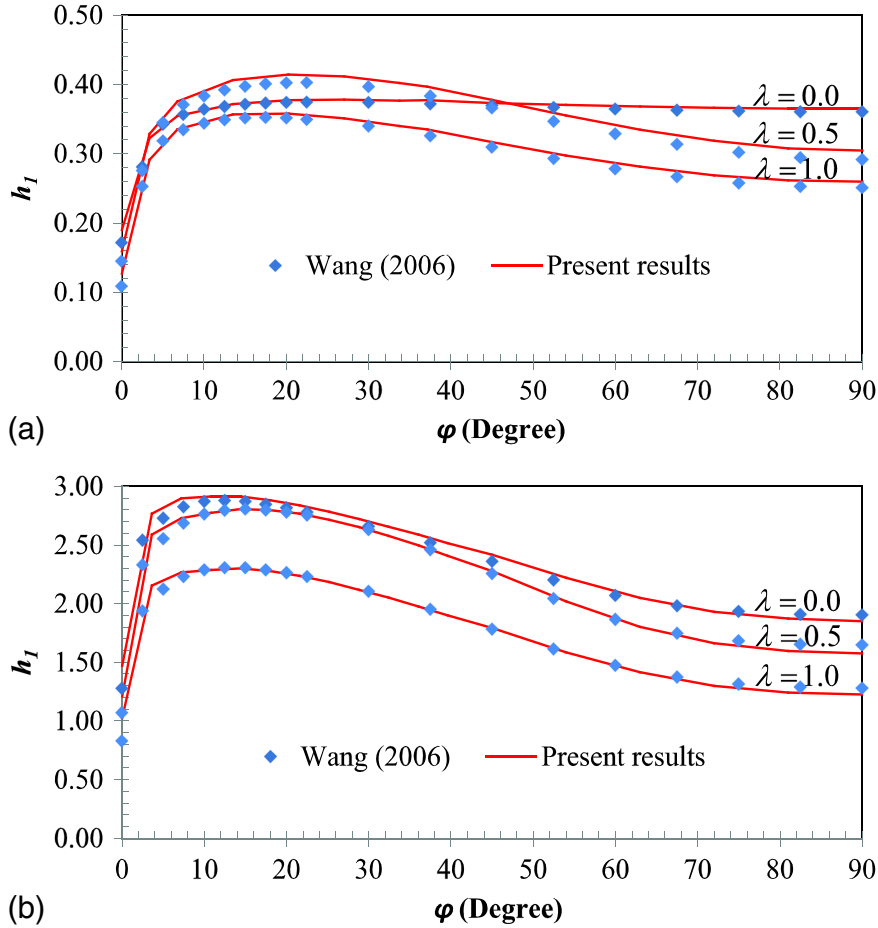

Fig. 3. Comparison of $h_{1}$ values from the present finite-element analyses and those from Wang (2006) $(n=3)$ : (a) $a / d=0.2$; (b) $a / d=0.8$

(1981) found that the results for the total $J$-integral calculated by adding up the elastic and fully plastic $J$-integrals are in good agreement with finite-element calculations and experimental results for the complete range of elastic-plastic deformation and materialhardening properties for different crack configurations. Therefore, the developed model is only indirectly verified by ensuring that the equivalent influence coefficients for the elastic $J$ integral, the normalized fully plastic $J$-integral, and total fracture toughness have been accurately determined.

For the equivalent influence coefficients, extensive verifications were conducted by $\mathrm{Li}$ et al. (2016) and Fu et al. (2017b) for the proposed finite-element models. Because the same model was used for the cracked plate with inclined surface cracks under biaxial loading, the accuracy of the calculated equivalent coefficients was assured. For fully plastic $J$-integrals, the models were verified by comparing the simulated results with those from an analytical solution for an embedded penny-shaped crack in an infinite body under far-field uniaxial and triaxial tension ( $\mathrm{Fu}$ et al. 2017a). In addition, the simulated results of $h_{1}$ for surface-cracked plates with inclination angle $\beta=0^{\circ}$ were compared with those from Wang (2006) as shown in Fig. 3. Good agreement was achieved for $a / d=0.2$ and 0.8 .

\section{Factors Affecting Elastic Fracture Toughness}

Using the developed model of Eq. (15) with influence coefficients $F_{e q}$ and normallized fully plastic $J$-integral $h_{1}$, the elastic fracture toughness $K_{C}^{e}$ of cracked plates under biaxial loading was determined. One of the benefits of the developed model is that the effects of key parameters can be quantitatively examined.

To take into account the effect of the biaxiality ratio on fracture toughness for Mode-I fracture, the experimental results for the lower bound of the total fracture toughness $K_{C}$ from Bass et al. (1996) 


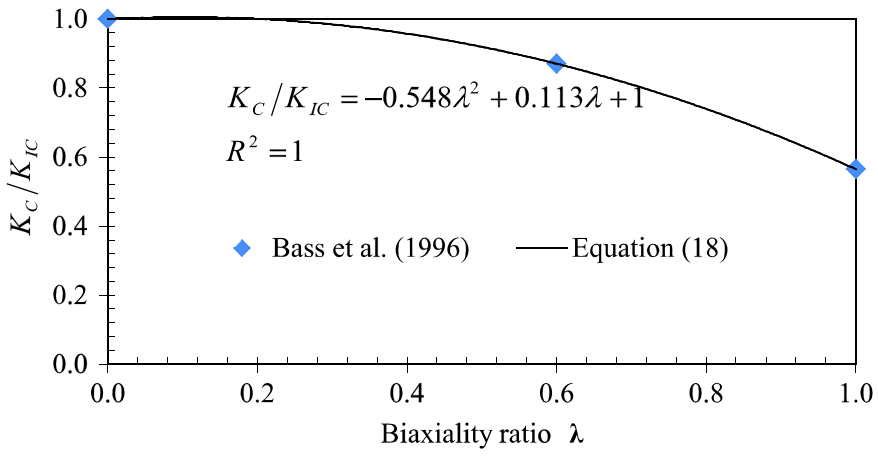

Fig. 4. Fracture toughness for Mode-I fracture with different biaxiality ratios

were employed and fitted to an equation using nonlinear regression as follows (Fig. 4):

$$
K_{C} / K_{I C}=-0.548 \lambda^{2}+0.113 \lambda+1
$$

For fracture toughness of mixed modes induced by inclined cracks, it is assumed that $K_{C} / K_{I C}$ for mixed Mode I and Mode II and for mixed Mode I and Mode III follows the trend of that in Pirondi and Dalle Donne (2001) and that in Raghavachary et al. (1990), respectively. The following formulas are obtained using nonlinear regression to respectively fit the two corresponding curves in Fig. 2:

$$
\begin{array}{ll}
K_{C} / K_{I C}=0.5043 M_{e}^{2}-0.1119 M_{e}+0.5160 & \text { Mixed mode I and II } \\
K_{C} / K_{I C}=0.4029 M_{e}^{2}-0.1735 M_{e}+0.7818 & \text { Mixed mode I and III }
\end{array}
$$

where $M_{e}$ ranges from 0 to 1 . For mixed-mode fractures, because of a lack of data on fracture toughness of Modes I, II, and III, the lowest mixed-mode fracture toughness from Eq. (22), $K_{C}=0.51 K_{I C}$, is employed in this section as an approximation.

\section{Effect of Yield Strength and Fracture Toughness}

Figs. 5 and 6 demonstrate the effect of material properties on elastic fracture toughness $K_{C}^{e}$ for a given cracked plate for $a / d=0.8$ and $n=3$ under uniaxial or biaxial loading along the entire crack front. Similar trends were found for other values of $a / d$ and $n$, but are omitted here.

Yield strength varies from 200 to $600 \mathrm{MPa}$ as shown in Fig. 5 whereas fracture toughness changes from 50 to $110 \mathrm{MPa} / \sqrt{\mathrm{m}}$ as shown in Fig. 6. It can be observed that for a given plate and crack geometry with a certain biaxiality ratio and strain-hardening coefficient, the larger the yield strength of the plate material, the greater portion of the brittle fracture the plate experiences and consequently the greater the relative elastic fracture toughness, defined as the ratio of elastic fracture toughness $K_{C}^{e}$ to total fracture toughness $K_{C}$. This makes sense from both the theoretical analysis and practical observation. Similarly, the greater the fracture toughness of the plate material, the greater portion of the plastic deformation the plate endures and hence the smaller the relative elastic fracture toughness. Again, this is consistent with practical experience.

\section{Effect of Biaxiality Ratio}

To investigate the effect of biaxiality ratio $\lambda$ on elastic fracture toughness $K_{C}^{e}$, a cracked plate with geometry $a / d=0.5$ and $n=$ 3 is employed for demonstration. From analyses, it is found that $\lambda$
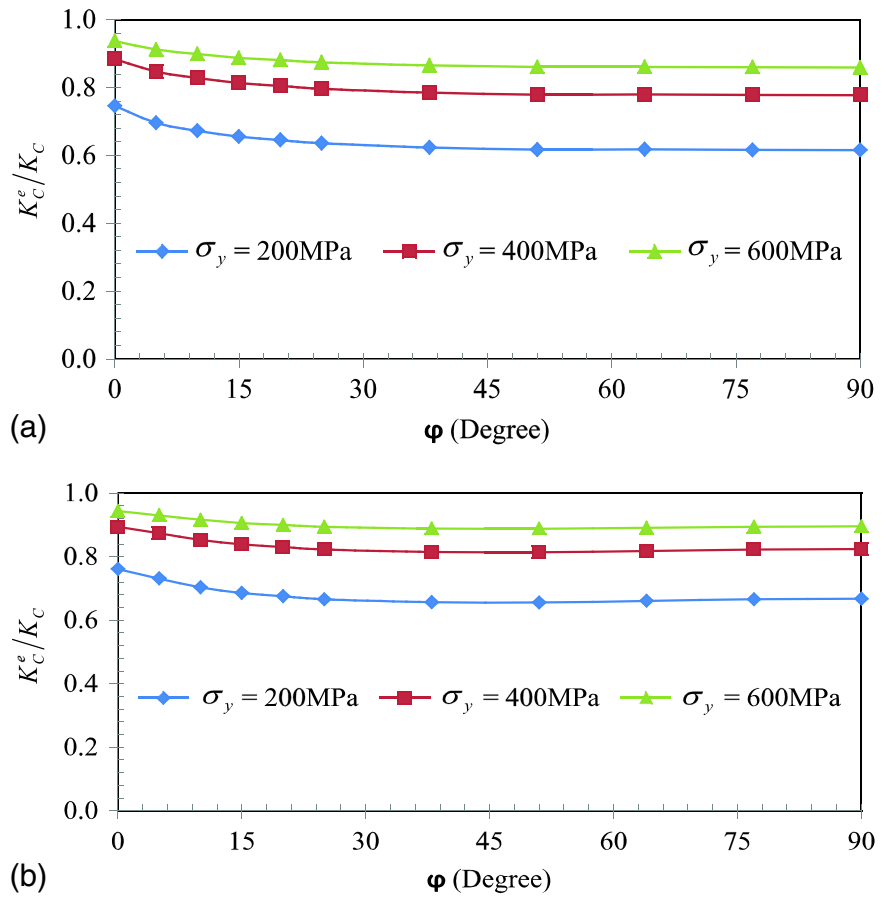

Fig. 5. Effect of yield strength on elastic fracture toughness along the crack front: (a) inclination angle $\beta=0^{\circ}, \lambda=0, a / d=0.8$, and $n=3$; (b) inclination angle $\beta=45^{\circ}, \lambda=1, a / d=0.8$, and $n=3$
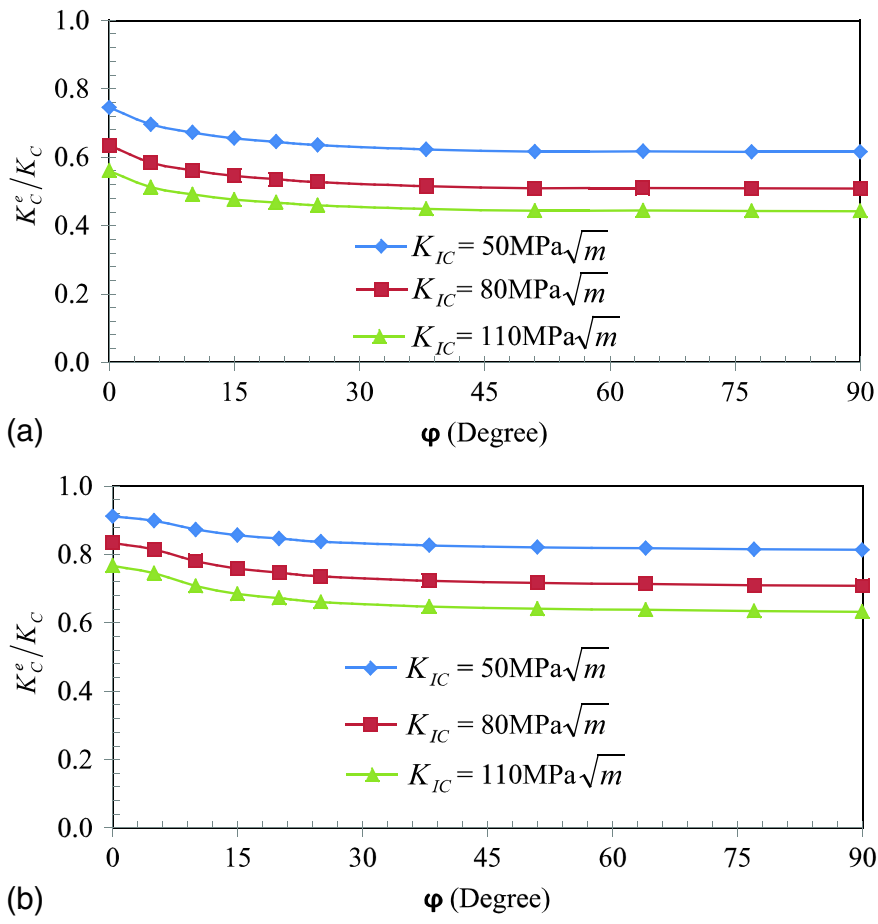

Fig. 6. Effect of Mode-I fracture toughness on elastic fracture toughness along the crack front: (a) inclination angle $\beta=0^{\circ}, \lambda=0$, $a / d=0.8$, and $n=3$; (b) inclination angle $\beta=45^{\circ}, \quad \lambda=1$, $a / d=0.8$, and $n=3$

does not have a significant effect on relative elastic fracture toughness if the total fracture toughness $K_{C}$ is assumed to be constant for different $\lambda$ as shown in Fig. 7(a) for $\beta=0^{\circ}, a / d=0.5$, and $n=3$. When $K_{C}$ follows Eq. (21) for different $\lambda$ values and takes the 

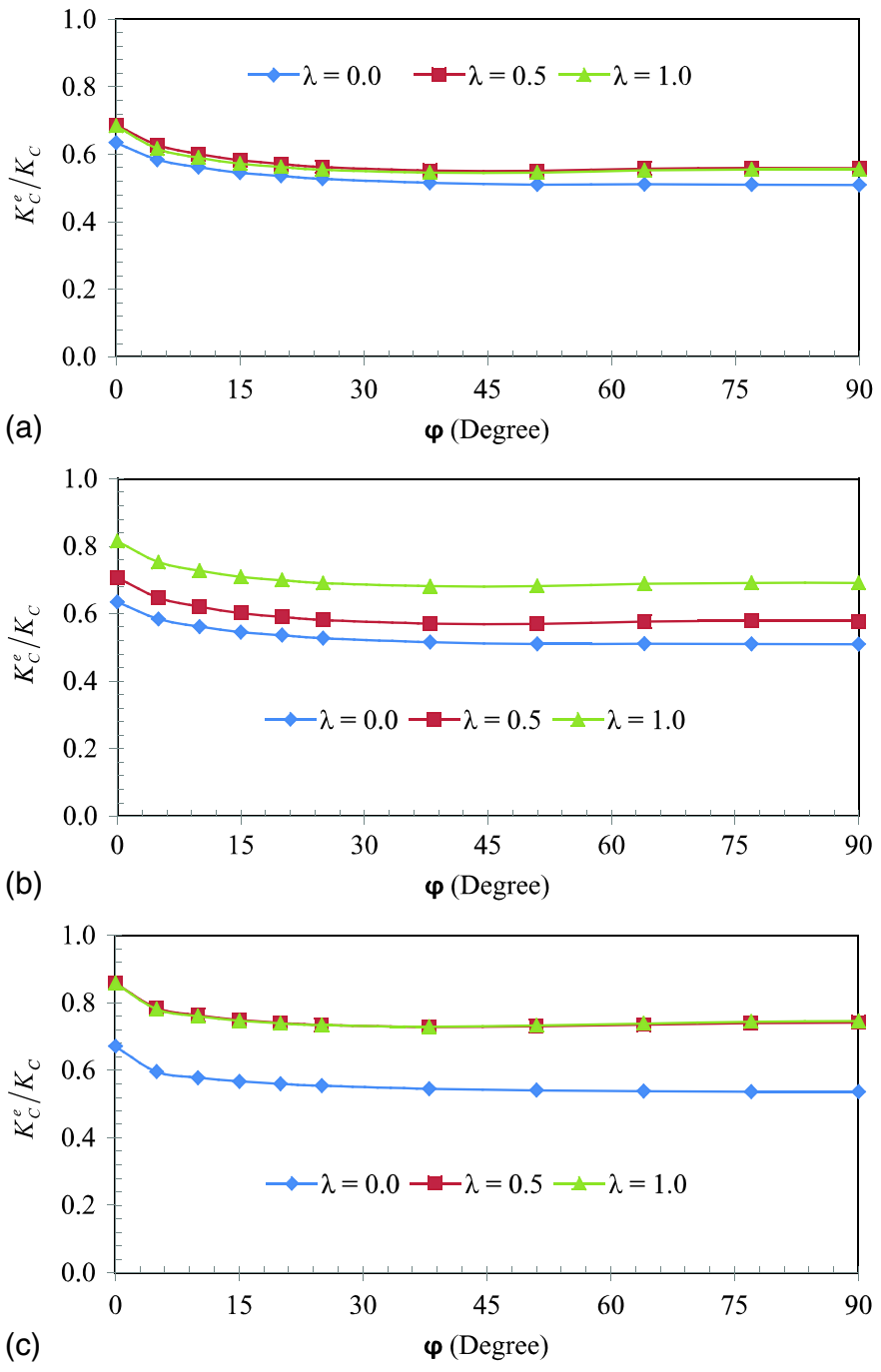

Fig. 7. Effect of biaxiality ratio on elastic fracture toughness: (a) inclination angle $\beta=0^{\circ}, a / d=0.5$, and $n=3$ with constant fracture toughness; (b) inclination angle $\beta=0^{\circ}, a / d=0.5$, and $n=3$ with different fracture toughness; (c) inclination angle $\beta=25^{\circ}$, $a / d=0.5$, and $n=3$ with different fracture toughness

minimum of Eq. (22) for different mode mixities, Figs. 7(b and c) show the effect that different $\lambda$ values have on relative elastic fracture toughness. Obvious differences are observed among the relative elastic fracture toughness values for different $\lambda$ values-in particular, $\lambda=0$ and $\lambda \neq 0$. Therefore, it can be concluded that the biaxiality ratio does affect relative elastic fracture toughness, mainly through its effect on the total fracture toughness $K_{C}$. This means that, if the biaxiality ratio does not affect total fracture toughness $K_{C}$ it does not have a significant influence on relative elastic fracture toughness.

\section{Effect of Inclination Angle}

The effects of crack inclination angle on elastic fracture toughness were also investigated. Fig. 8 shows how relative elastic fracture toughness changes with different inclination angles along the entire crack front for $\lambda=0, a / d=0.5$, and $n=3$ and the same total fracture toughness for different inclination angles. It can be observed that relative elastic fracture toughness decreases with increasing inclination angles from $0^{\circ}$ to $45^{\circ}$. This means that the
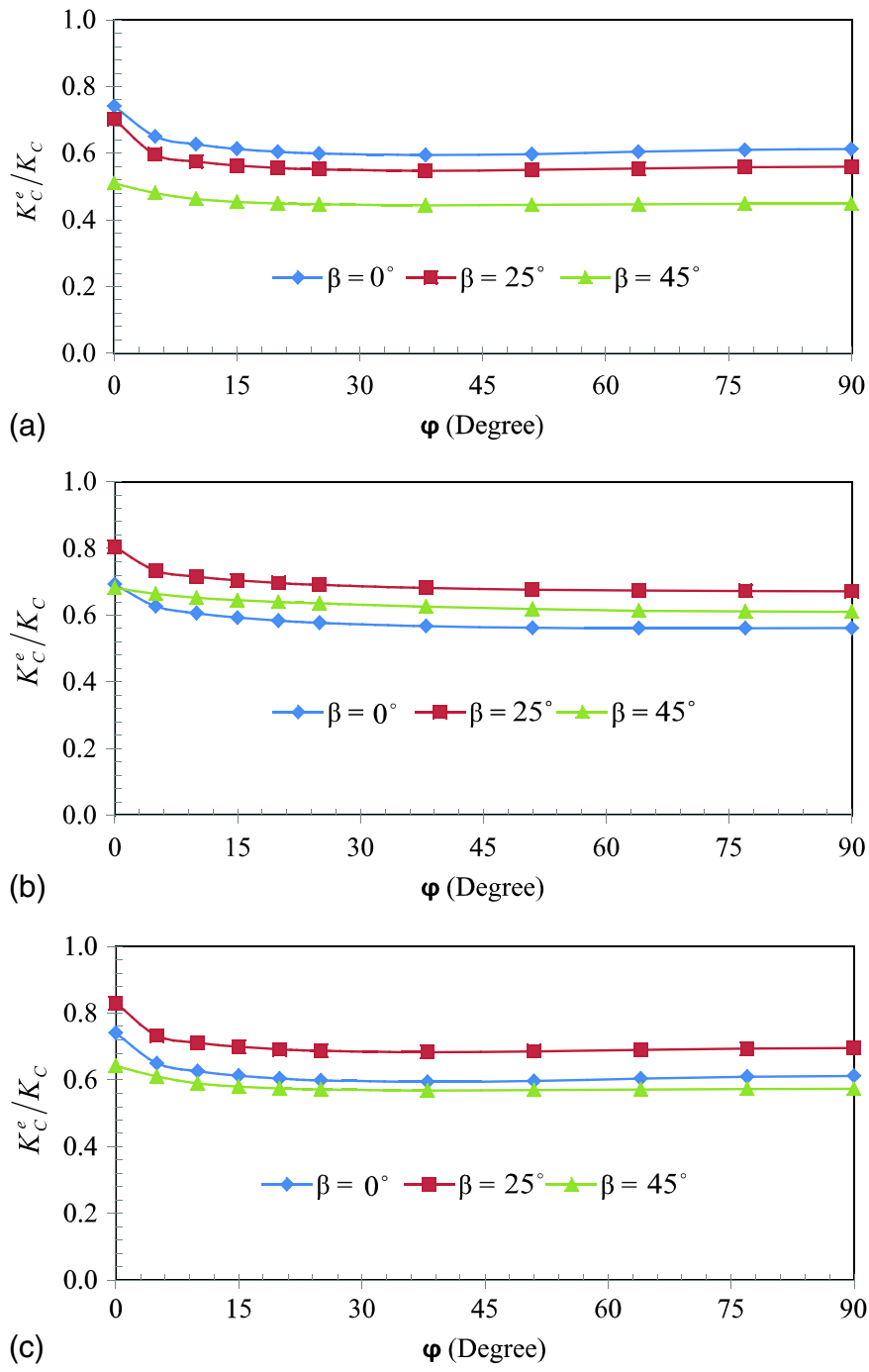

Fig. 8. Effect of inclination angle on elastic fracture toughness: (a) biaxiality ratio $\lambda=0, a / d=0.5$, and $n=3$ with constant fracture toughness; (b) biaxiality ratio $\lambda=0, a / d=0.5$, and $n=3$ with different fracture toughness; (c) biaxiality ratio $\lambda=1, a / d=0.5$, and $n=3$ with different fracture toughness

larger the inclination angle, the more plastic deformation that develops in the plate. This makes sense because, for a given crack size, the larger the inclination angle, the more difficult it is for plates to fail in a brittle way. However, this trend changes when reduction of fracture toughness is considered for mixed-mode fracture. Figs. 8(b and c) show the effect of inclination angle on relative elastic fracture toughness assuming $K_{C}=0.51 K_{I C}$ for mixedmode fracture. No general trend can be discerned because reducing total fracture toughness increases relative elastic fracture toughness whereas reducing the inclination angle decreases it. Therefore, the trend actually depends on the amount of reduction in fracture toughness and the increment of inclination angles.

\section{Effect of Relative Crack Depth}

The effects of relative crack depth $a / d$ on $K_{C}^{e}$ of cracked plates are investigated by considering relative depth ratios $a / d$ of $0.2,0.5$, and 0.8. Two cases are shown in Fig. 9. Other cases can be produced in a similar way, but they are omitted here. It can be seen that the plate material demonstrates more brittleness as the relative 

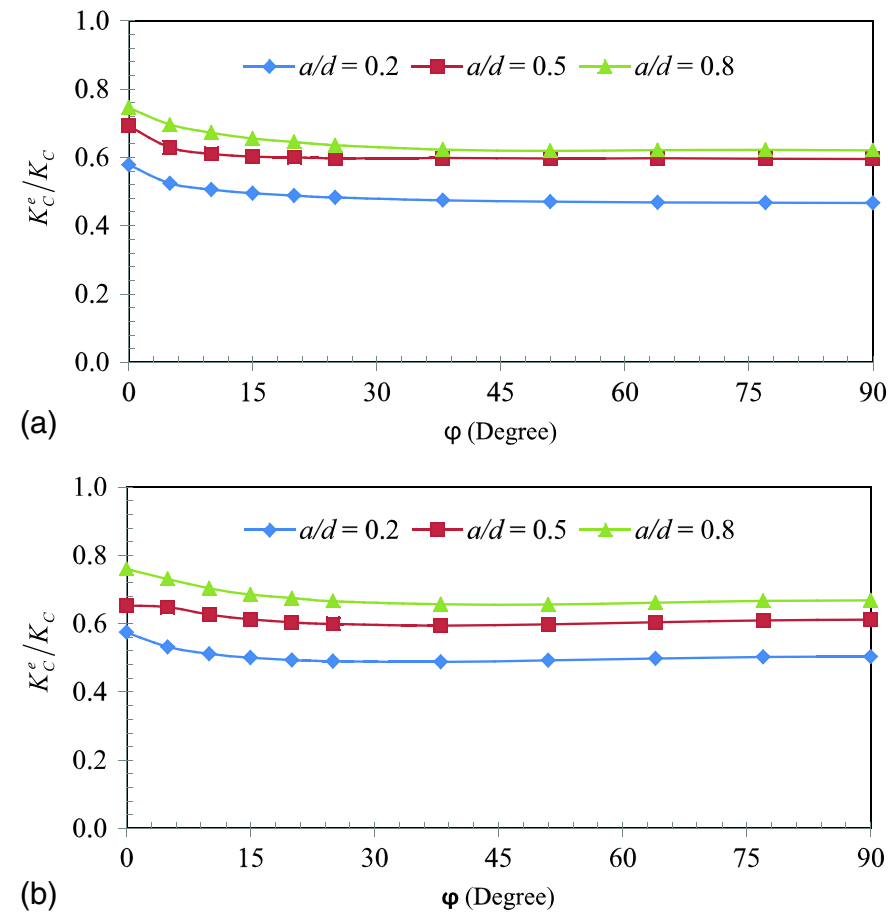

Fig. 9. Effect of relative crack depth on elastic fracture toughness along the crack front: (a) inclination angle $\beta=0^{\circ}, \lambda=0$, and $n=3$; (b) inclination angle $\beta=45^{\circ}, \lambda=1$, and $n=3$

depth $a / d$ increases from 0.2 to 0.8 , resulting in larger values of relative elastic fracture toughness. This means that a larger $a / d$ ratio leads to less plastic deformation developing around the crack, which makes sense because greater relative depth, which means less material in the remaining ligament, results in a plate with a lower level of plasticity compared with less relative depth.

\section{Conclusions}

The $J$-integral solution was derived to determine elastic fracture toughness for ductile metal plates with inclined surface cracks under biaxial loading. The derived elastic fracture toughness is a function of plate and crack geometry, strain-hardening coefficient, yield strength, fracture toughness, biaxiality ratio, and inclination angle. Parametric studies have shown that an increase in yield strength or relative crack depth, or a decrease in Mode-I fracture toughness, leads to greater relative elastic fracture toughness. It has also been shown that the effect of biaxiality ratio and inclination angle on elastic fracture toughness is highly dependent on total fracture toughness values, which highlights the need for accurate experimental determination of total fracture toughness taking into account the effect of biaxial and mixed-mode loadings. It can be concluded that the developed elastic fracture toughness model enables engineers and asset managers to accurately predict fracture failure of ductile thin metal structures with inclined cracks under biaxial loading.

\section{Acknowledgments}

Financial support from the Australian Research Council under DP140101547, LP150100413, and DP170102211 is gratefully acknowledged.

\section{References}

ABAQUS version 6.11 [Computer software]. Dassault Systèmes Simulia Corp., Providence, RI.

Anderson, T. L. (1991). Fracture mechanics: Fundamentals and applications, CRC Press, Boca Raton, FL.

Anderson, T. L. (2005). Fracture mechanics: Fundamentals and applications, 3rd Ed., CRC Press, Boca Raton, FL.

Bass, B. R., Bryson, J. W., Dickson, T. L., McAfee, W. J., and Pennell, W. E. (1996). "Development of a shallow flaw fracture assessment methodology for nuclear reactor pressure vessels." Proc., 3rd Int. Conf. on Engineering Structural Integrity Assessment, Churchill College, Cambridge, U.K.

Bass, B. R., Shum, D. K., and Keeney-Walker, J. (1992). "Constraint effects on fracture toughness for circumferentially oriented cracks in reactor pressure vessels." NUREG/CR-6008, ORNL/TM-12131, Oak Ridge National Laboratory, Oak Ridge, TN.

Fu, G. Y., Yang, W., and Li, C. Q. (2017a). "Elastic and fully plastic J-integrals for mixed mode fracture induced by inclined cracks in plates under biaxial loading." Eng. Fract. Mech., 186, 483-495.

Fu, G. Y., Yang, W., and Li, C. Q. (2017b). "Stress intensity factors for mixed mode fracture induced by inclined cracks in pipes under axial tension and bending." Theor. Appl. Fract. Mech., 89, 100-109.

Green, A. E., and Sneddon, I. N. (1950). "The distribution of stress in the neighbourhood of a flat elliptical crack in an elastic solid." Math. Proc. Cambridge Philos. Soc., 46(01), 159-163.

Hallbäck, N. (1997). "Mixed-mode I/II fracture behavior of a high strength steel.” Int. J. Fract., 87(4), 363-388.

Hutchinson, J. W. (1968). "Singular behavior at the end of a tensile crack tip in a hardening material." J. Mech. Phys. Solids, 16(1), 13-31.

Jansson, S. (1986). "Fully plastic plane stress solutions for biaxially loaded centre cracked plates." ASME J. Appl. Mech., 53(3), 555-560.

Jones, D. L., Poulose, P. K., and Liebowitz, H. (1986). "The effects of biaxial loading on the fracture characteristics of several engineering materials." Eng. Fract. Mech., 24(2), 187-205.

Kamat, A. M., Hirth, J. P., Hoagland, R., and Feng, X. X. (1994). "A suggested test procedure to measure mixed mode I-III fracture toughness of brittle materials." J. Test. Eval., 22(4), 327-334.

Kamat, S. V., and Hirth, J. P. (1995). "Mixed mode fracture toughness of engineering materials." J. Eng. Mater. Technol., 117(4), 391-394.

Kamat, S. V., and Hirth, J. P. (1996). "Mixed mode I/II fracture toughness of 2034 aluminum alloys." Acta Materialia, 44(1), 201-208.

Keiichiro, T., and Hitoshi, I. (1992). "Elastic-plastic fracture toughness test under mixed mode I-II loading." Eng. Fract. Mech., 41(4), 529-540.

Kumar, V., German, M. D., and Shih, C. F. (1981). "An engineering approach for elastic plastic fracture analysis." Tropical Rep., NP-1931, Research Project 1237-1, General Electric Company, Boston.

Li, C. Q., Fu, G. Y., and Yang, W. (2016). "Stress intensity factors for inclined external surface cracks in pressurised pipes." Eng. Fract. Mech., $165,72-86$.

Li, C. Q., Fu, G. Y., Yang, W., and Yang, S. T. (2017). "Derivation of elastic fracture toughness for ductile metal pipes with circumferential external cracks under combined tension and bending." Eng. Fract. Mech., 178, $39-49$.

Liu, S., Chao, Y. J., and Zhu, X. (2004). “Tensile-shear transition in mixed mode I/III fracture." Int. J. Solids Struct., 41(22-23), 6147-6172.

Manoharan, M., Hirth, J. P., and Rosenfield, A. R. (1990). "A suggested procedure for combined mode I-mode III fracture toughness testing." J. Test. Eval., 18(2), 106-114.

Milne, I., Ainsworth, R. A., Dowling, A. R., and Stewart, A. T. (1988). "Assessment of the integrity of structures containing defects." Int. J. Press. Vessels Pip., 32(1-4), 3-104.

Mostafavi, M., Smith, D. J., and Pavier, M. J. (2011). "Fracture of aluminium alloy 2024 under biaxial and triaxial loading." Eng. Fract. Mech., 78(8), 1705-1716.

Paradkar, A. G., and Kamat, S. V. (2011). "Fracture toughness of Ti-15Al$8 \mathrm{Nb}$ alloy under mixed mode I/III loading." Mater. Sci. Eng., 528(9), 3283-3288.

Pirondi, A., and Dalle Donne, C. (2001). "Characterization of ductile mixed-mode fracture with the crack-tip displacement vector." Eng. Fract. Mech., 68(12), 1385-1402.

Pook, L. P. (1995). “On fatigue crack paths.” Int. J. Fatigue, 17(1), 5-13. 
Raghavachary, S., Rosenfield, A. R., and Hirth, J. P. (1990). "Mixed mode I/III fracture toughness of an experimental rotor steel." Metall. Trans. A, 21A(9), 2539-2545.

Ramberg, W., and Osgood, W. R. (1943). "Description of stress-strain curves by three parameters." Technical Note No. 902, National Advisory Committee for Aeronautics, Washington DC.

Rice, J. R. (1968). "A path independent integral and the approximate analysis of strain concentration by notches and cracks." J. Appl. Mech., 35(2), 379-386.

Rice, J. R., and Rosengren, G. F. (1968). "Plane strain deformation near a crack tip in a power-law hardening material." J. Mech. Phys. Solids, 16(1), 1-12.

Richard, H. A., Eberlein, A., and Schirmeisen, N. H. (2013). "Experiments on cracks under spatial loading." 13th Int. Conf. on Fracture, Curran Associate, Inc., New York.

Richard, H. A., and Kuna, M. (1990). "Theoretical and experimental study of superimposed fracture modes I, II, and III." Eng. Fract. Mech., 35(6), 949-960.

Saka, M., and Tanaka, S. (1986). "Stain and stress fields near the blunted tip of a crack under mixed mode loading and the implication for fracture." Mech. Mater., 5(4), 331-338.

Shih, C. F. (1974). "Small-scale yielding analysis of mixed mode plane strain crack problem." ASTM STP 560, ASTM, West Conshohocken, PA, 187-210.

Shiratori, M., Niyoshi, T., and Tanikawa, K. (1987). "Analysis of stress intensity factors for surface cracks subjected to arbitrarily distributed surface cracks." Stress intensity factors handbook, Y. Murakami, ed., Pergamon, Tokyo, 698-727.

Sih, C. F. (1976). "J-integral estimates for strain hardening materials in antiplane shear using fully plastic solution." ASTM STP 590, ASTM, West Conshohocken, PA, 3-26.

SINTAP (Structural INTegrity Assessment Procedures for European Industry). (1999). "Structural integrity assessment procedures for European industry-final procedure." European Union Project No. BE95-1426, Europe.

Wang, X. (2006). "Fully plastic J-integral solutions for surface cracked plates under biaxial loading." Eng. Fract. Mech., 73(11), 1581-1595.

Yang, S. T., Li, C. Q., and Yang, W. (2016). "Analytical model of elastic fracture toughness for steel pipes with internal cracks." Eng. Fract. Mech., 153, 50-60.

Yang, W., Fu, G. Y., and Li, C. Q. (2017). "Elastic fracture toughness of ductile materials." J. Eng. Mech., 10.1061/(ASCE)EM.1943-7889 $.0001321,04017111$.

Zhu, X. K., and Joyce, J. A. (2012). "Review of fracture toughness (G, K, J, CTOD, CTOA) testing and standardization." Eng. Fract. Mech., 85, $1-46$. 